\title{
Impedance spectroscopy of $\mathrm{Ba}_{3} \mathrm{Sr}_{2} \mathrm{DyTi}_{3} \mathrm{~V}_{7} \mathrm{O}_{30}$ ceramic
}

\author{
P S SAHOO ${ }^{\dagger}$, A PANIGRAHI ${ }^{\dagger \dagger}$, S K PATRI and R N P CHOUDHARY* \\ Department of Physics and Meteorology, Indian Institute of Technology, Kharagpur 721 302, India \\ ${ }^{\dagger}$ Department of Physics, Betnoti College, Betnoti 757 025, India \\ ${ }^{\dagger \dagger}$ Department of Physics, D.N. College, Itanagar 791 111, India
}

MS received 25 December 2008; revised 5 June 2009

\begin{abstract}
Polycrystalline sample of $\mathrm{Ba}_{3} \mathrm{Sr}_{2} \mathrm{DyTi}_{3} \mathrm{~V}_{7} \mathrm{O}_{30}$ was prepared at $950^{\circ} \mathrm{C}$ using a high-temperature solid-state reaction technique. $\mathrm{X}$-ray structural analysis indicated the formation of a single-phase orthorhombic structure with lattice parameters: $a=12.2719$ (39) $\AA, b=8.9715(39) \AA$ and $c=19.7812(39) \AA$. Microstructural study showed densely packed uniform distribution of grains over the surface of the sample. The a.c. impedance plots were used as tools to analyse the electrical response of the sample as a function of frequency at different temperatures $\left(30-500^{\circ} \mathrm{C}\right)$. These plots revealed the presence of grain boundary effect, from $200^{\circ} \mathrm{C}$ onwards. Complex impedance analysis showed non-Debye type of dielectric relaxation. The Nyquist plots showed the negative temperature coefficient of resistance character of $\mathrm{Ba}_{3} \mathrm{Sr}_{2} \mathrm{DyTi}_{3} \mathrm{~V}_{7} \mathrm{O}_{30}$. A hopping mechanism of electrical transport processes in the system is evident from the modulus analysis. The activation energy of the compound (calculated both from loss and modulus spectrum) is the same, and hence the relaxation process may be attributed to the same type of charge carrier.
\end{abstract}

Keywords. Ceramics; X-ray diffraction; dielectric properties; electrical conductivity.

\section{Introduction}

Though a large number of ferroelectric oxides of different structural families (i.e. perovskite, tungsten-bronze, spinel, etc) are now available, some compounds of perovskite and tungsten-bronze (TB) families (Stenger and Burggraaf 1980; Singh et al 1992) in pure and/or complex form, have been found to be very important because of their various physical properties suitable for the fabrication of devices. Till date most of these materials are lead based compounds (i.e. lead titanate $\left(\mathrm{PbTiO}_{3}\right)$, lead zirconate titanate $\left(\mathrm{PbZr}_{1-x} \mathrm{Ti}_{x} \mathrm{O}_{3}\right)$, lead magnesium niobate $\left(\mathrm{PbMg}_{1 / 3}\right.$ $\mathrm{Nb}_{2 / 3} \mathrm{O}_{3}$ ), etc) which are toxic, hazardous and also the $\mathrm{Pb}$-based gadgets are not recyclable. So in this 'no-lead or low-lead era' the search for alternative materials for potential applications has now become a focal theme of the present day research. Taking into consideration the environmental, health and social aspects, manufacturers have been constrained to reduce and ultimately eliminate the $\mathrm{Pb}$-content of the materials. Hence, the search for alternative materials for multilayer capacitors (MLCs), piezoelectric/pyroelectric applications has now become a focal theme of the present day research. The low temperature behaviour of titanate-based materials is often controlled by grain boundaries, and therefore, the knowledge of behaviour of grain boundary is important. Complex impedance spectroscopic technique is considered to be a

\footnotetext{
*Author for correspondence (crnpfl@phy.iitkgp.ernet.in)
}

promising non-destructive testing method for analysing the electrical processes occurring in a compound on the application of a.c. signal as input perturbation. The output response of polycrystalline compound (when plotted in a complex plane) represents grain, grain boundary and electrode properties with different time constants leading to successive semicircles. The properties of tungstenbronze structure could vary with different elements, and hence it becomes complicated due to their complexity in crystal structure (Jiang et al 2005). In this paper, we have analysed the impedance properties of $\mathrm{Ba}_{3} \mathrm{Sr}_{2} \mathrm{DyTi}_{3} \mathrm{~V}_{7} \mathrm{O}_{30}$.

\section{Experimental}

The polycrystalline sample of $\mathrm{Ba}_{3} \mathrm{Sr}_{2} \mathrm{DyTi}_{3} \mathrm{~V}_{7} \mathrm{O}_{30}$ (BSDTV) was prepared by a mixed oxide route using high purity (99.9\%) ingredients; $\mathrm{BaCO}_{3}$ (M/s Sarabhai M. Chemicals Pvt. Ltd., India), $\mathrm{SrCO}_{3}(\mathrm{M} / \mathrm{s}$ Sarabhai M. Chemicals Pvt. Ltd., India), $\mathrm{TiO}_{2}$ (M/s Sarabhai M. Chemicals Pvt. Ltd., India), $\mathrm{Dy}_{2} \mathrm{O}_{3}$ (M/s Sarabhai M. Chemicals Pvt. Ltd., India), and $\mathrm{V}_{2} \mathrm{O}_{5}(\mathrm{M} / \mathrm{s}$ Koch Light Ltd., England) in a suitable stoichiometry. These ingredients were thoroughly mixed and ground in dry (air) and wet (methanol) medium for $2 \mathrm{~h}$ each in an agate mortar. The homogeneous mixture of the compound was heated in an alumina crucible starting from $800^{\circ} \mathrm{C}$ in steps of $50^{\circ} \mathrm{C}$. The process of grinding and calcination was thus repeated several times until the formation of the desired compound was confirmed with $950^{\circ} \mathrm{C}$ heating for $6 \mathrm{~h}$. A small amount of 
polyvinyl alcohol was added to the calcined powder for the fabrication of pellets, which was burnt out during high-temperature sintering. The circular disc shaped pellets was prepared by applying a uniaxial pressure of $5 \times 10^{6} \mathrm{~N} / \mathrm{m}^{2}$. The pellets were subsequently sintered at an optimized temperature of $1000^{\circ} \mathrm{C}$ for $12 \mathrm{~h}$. A preliminary study on compound formation and structural parameters was carried out using an X-ray diffraction (XRD) technique with an X-ray powder diffractometer (Rigaku Miniflex, Japan). The XRD pattern of the calcined powder was recorded at room temperature with $\mathrm{CuK}_{\alpha}$ radiation $(1.5405 \AA)$ in a wide range of Bragg's angles $2 \theta$ $\left(20^{\circ} \leq 2 \theta \leq 80^{\circ}\right)$ at a scan speed of $4^{\circ} / \mathrm{min}$. Microstructure of a sintered pellet was recorded by JEOL JSM-5800 scanning electron microscope (SEM). The pellet was then electroded with high purity air-drying silver paste and then dried at $150^{\circ} \mathrm{C}$ for $4 \mathrm{~h}$. Impedance spectroscopic analysis was done using a computer-controlled impedance analyser (PSM 1735, model: $\mathrm{N} 4 \mathrm{~L}$ ) over a wide range of temperature $\left(30-500^{\circ} \mathrm{C}\right)$ and frequency $\left(10^{3}-10^{6} \mathrm{~Hz}\right)$.

\section{Results and discussion}

\subsection{Structural analysis}

Figure 1 shows the XRD pattern of the BSDTV sample. All the reflection peaks are found to be sharp and single, which are different from those of ingredients. This confirms the formation of new single-phase compound. All the peaks were indexed taking their $2 \theta$ values using a computer program package, 'POWDMULT' [Powdmult] in different crystal systems and cell configurations. On the basis of the best agreement (based on least-squares refinement) between observed (obs) and calculated (cal) interplaner distance $d$ (i.e. $\sum\left(d_{\mathrm{obs}}-d_{\mathrm{cal}}\right)=$ minimum), an orthorhombic unit cell was selected with lattice parameters: $a=12.2719(39) \AA, b=8.9715(39) \AA, c=19.7812(39) \AA$ (estimated standard deviation in parenthesis) which are consistent with the reported ones (Ranga Raju and Choudhary 2003; Sahoo et al 2008a). The coherently scattered crystallite size $(D)$ of the compound was determined using Scherrer's equation: $D=0.89 \lambda /\left(\beta_{1 / 2} \cos \theta_{h k l}\right)$, where $\lambda=1.5405 \AA$ and $\beta_{1 / 2}=$ peak width of the reflection at half maxima (Klug 1974). The average crystallite size calculated from Scherrer's equation was $\sim 16 \mathrm{~nm}$.

\subsection{Microstructural analysis}

The SEM micrograph of the BSSTV compound at room temperature is shown in figure 1 (inset). The grain size evaluated from the micrograph is in the range of $1-2 \mu \mathrm{m}$. It was found that the grains were homogenously and uniformly distributed over the entire surface of the sample. An expected grain size of the sample obtained here is much larger compared to the crystallite size calculated from Scherrer equation, which implied that single grain has several crystallites (Choudhary et al 2006).

\subsection{Impedance analysis}

The complex impedance spectroscopy (CIS) technique (MacDonald 1987) was used to analyse the electrical response (i.e. transport properties) of a polycrystalline sample in a wide range of frequency $\left(10^{3}-10^{6} \mathrm{~Hz}\right)$ at different temperatures $\left(30-500^{\circ} \mathrm{C}\right)$. The ferroelectric phase transition temperature of this material was found at $326^{\circ} \mathrm{C}$ (Sahoo et al 2008b). Figures 2(a) and (b) show the variation of the real part $\left(Z^{\prime}\right)$ and imaginary part $\left(Z^{\prime \prime}\right)$ of impedance with frequency at various temperatures, respectively. It is observed that the magnitude of $Z^{\prime}$ decreases with the increase in both frequency as well as temperature indicating an increase in a.c. conductivity with the rise in temperature and frequency. The values of $Z^{\prime}$ for all temperatures merge above $10 \mathrm{kHz}$. This may be due to the release of space charges as a result of reduction in the barrier properties of material with the rise in temperature, and may be a responsible factor for the enhancement of a.c. conductivity of material with temperature at higher frequencies. Further, at low frequencies the value of $Z^{\prime}$ decreases with rise in temperature showing negative temperature coefficient of resistance (NTCR) type behaviour (like that of semiconductors). The curves show that the $Z^{\prime \prime}$ values reach a maxima peak $\left(Z_{\max }^{\prime \prime}\right)$ above $225^{\circ} \mathrm{C}$. The value of $Z_{\max }^{\prime \prime}$ shifts to higher frequencies on increasing temperature. A typical peak broadening, which is slightly asymmetrical in nature, can be observed with the rise in temperature. The broadening of peaks (explicit plots of $Z^{\prime \prime}$ ) suggests that, there is a spread of relaxation time (i.e. the existence of a temperature dependent electrical relaxation phenomenon in the mate-

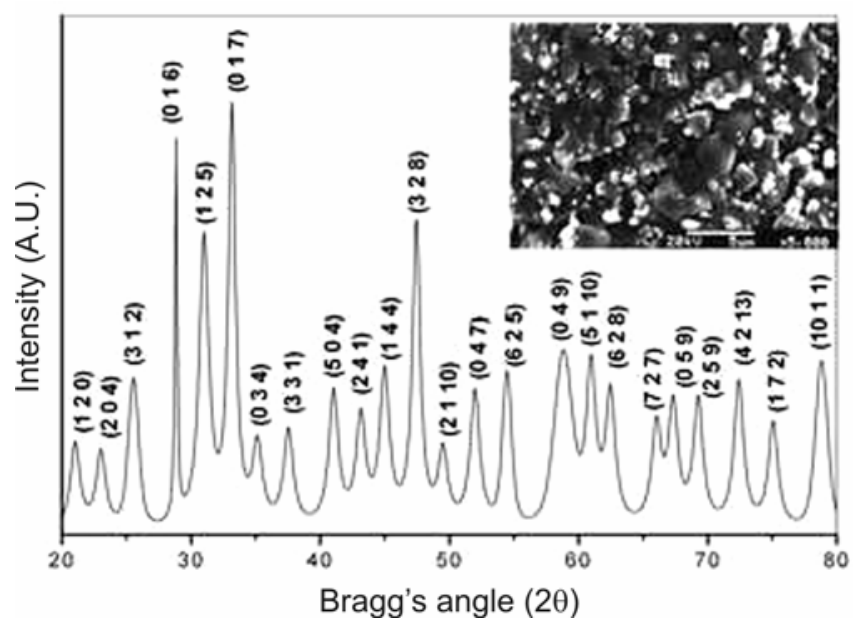

Figure 1. Room temperature XRD pattern and SEM micrograph (inset) of $\mathrm{Ba}_{3} \mathrm{Sr}_{2} \mathrm{DyTi}_{3} \mathrm{~V}_{7} \mathrm{O}_{30}$. 
rial (Suman et al 2005)). The merging of $Z$ " values below $T_{\mathrm{c}}$ (i.e. $\leq 326^{\circ} \mathrm{C}$ ) in the high frequency region may possibly be an indication of the accumulation of space charge in the material. Above $T_{\mathrm{c}}$, the curves merge in the lower frequency region. The peaks shift toward high frequency region with rise in temperature. For the temperatures below $225^{\circ} \mathrm{C}$, the peaks were beyond the range of frequency measurement.

Figure 3 shows a set of impedance data taken over a wide frequency range at several temperatures as a Nyquist diagram (complex impedance spectrum). It is observed that with the increase in temperature, the slope of the lines decreases and their curve bends towards real $\left(Z^{\prime}\right)$ axis, and thus at temperature $325^{\circ} \mathrm{C}$, a semicircle could be traced, indicating the increase in conductivity of the sample. The centre of the semicircular arc shifts towards the origin on increasing temperature which indicates that the conductivity of the samples increases with
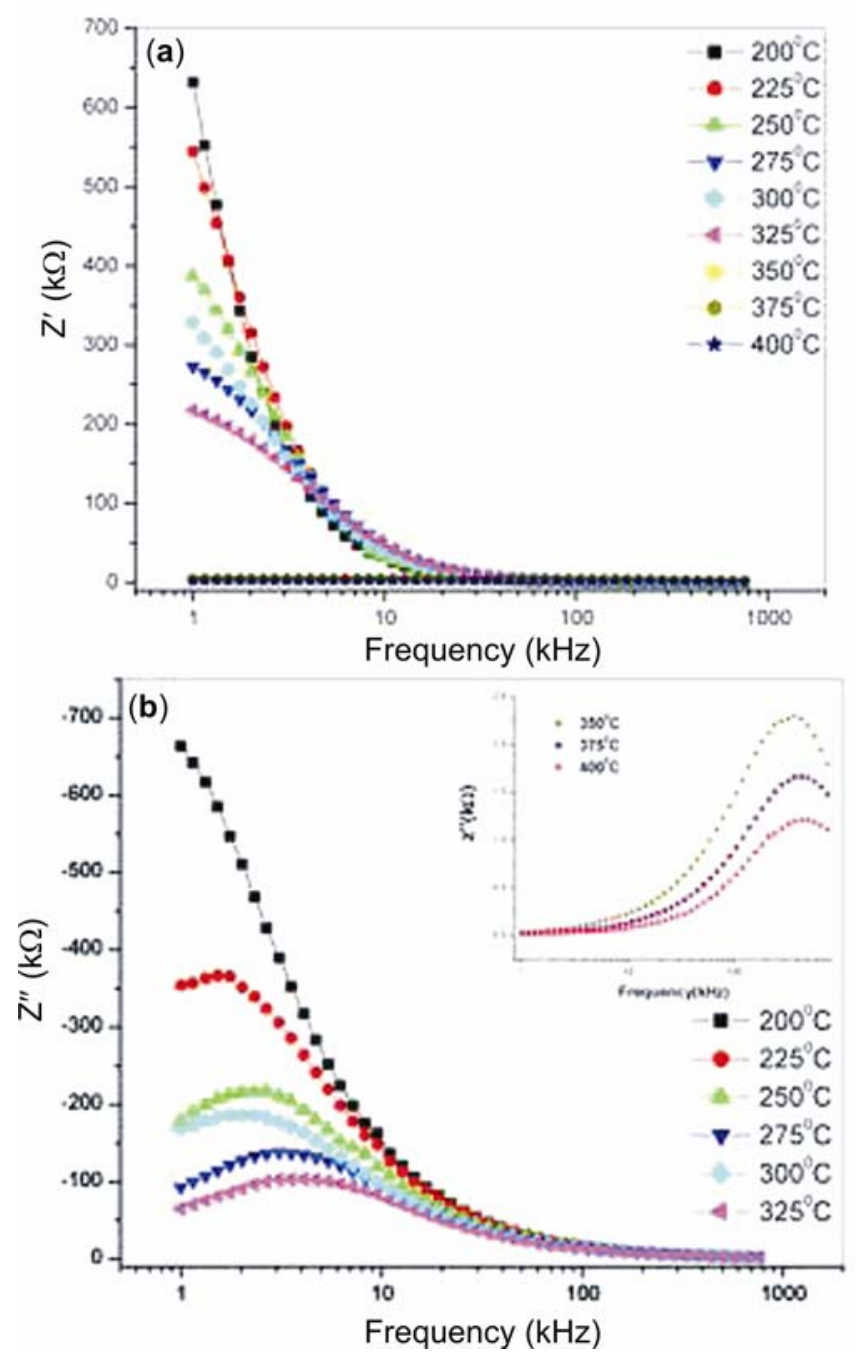

Figure 2. Variation of (a) real part of complex impedance $\left(Z^{\prime}\right)$ and (b) imaginary part of complex impedance $\left(Z^{\prime \prime}\right)$ of $\mathrm{Ba}_{3} \mathrm{Sr}_{2} \mathrm{DyTi}_{3} \mathrm{~V}_{7} \mathrm{O}_{30}$ with frequency. increase in temperature. In this material, dipolar relaxation process occurs in the material since semicircular arcs are observed in the high-frequency zone. Single semicircular arcs observed in the complex plane at elevated temperatures reveal that the conduction in the material is predominant in grains rather than in grain boundaries. In order to confirm the ambiguity arising due to the presence of grain/grain boundary effect (Choudhary et al 2007) at elevated temperatures, the impedance data have been re-plotted in the modulus formalism at selected temperatures as shown in figure 4 . It is clear that the modulus plane plots two semicircles; the intercept of first semicircle on the real axis indicates the total capacitance contributed by the grain and that of second semicircle by grain boundary. The modulus spectra show a marked

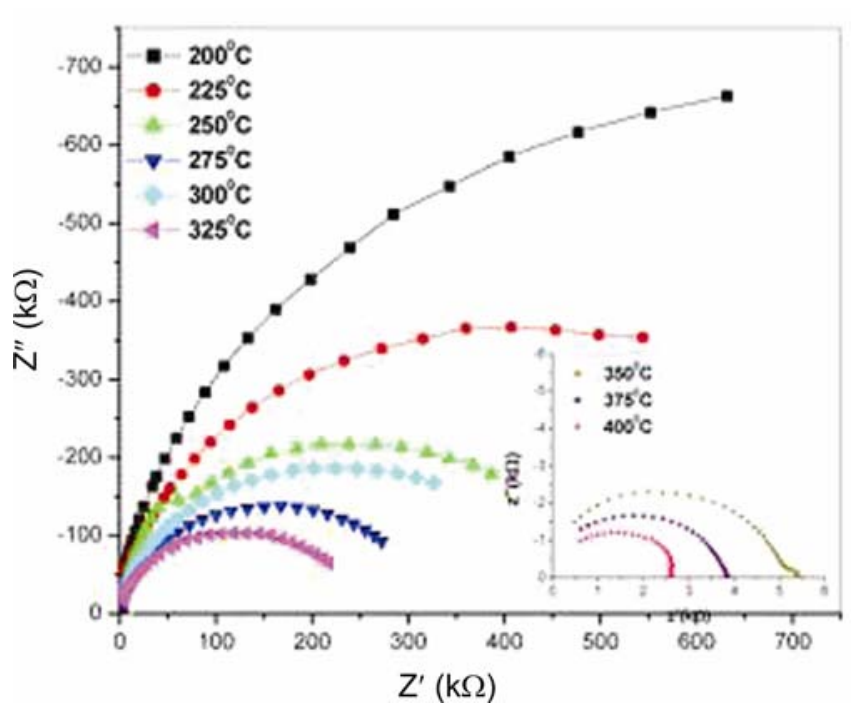

Figure 3. Nyquist plot of $\mathrm{Ba}_{3} \mathrm{Sr}_{2} \mathrm{DyTi}_{3} \mathrm{~V}_{7} \mathrm{O}_{30}$.

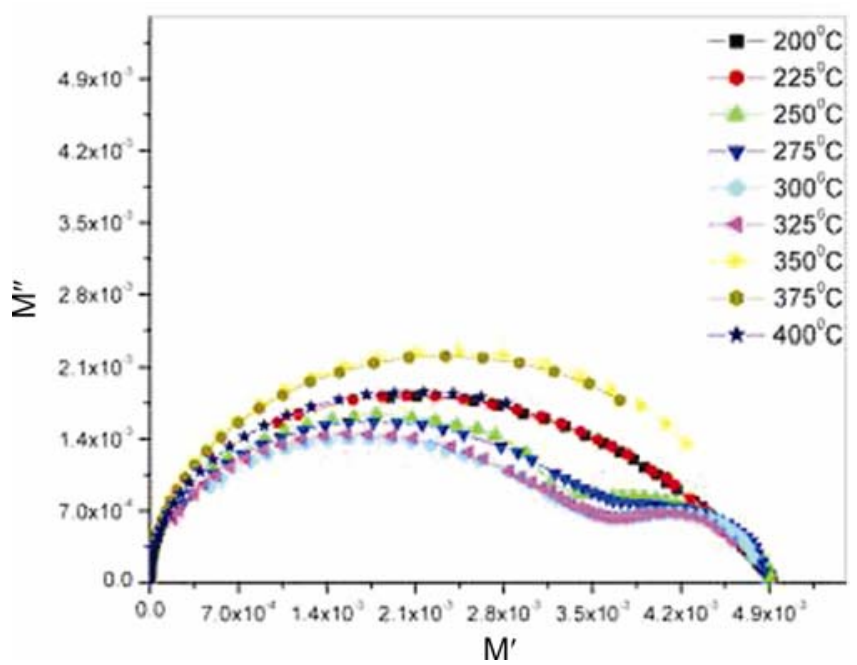

Figure 4. Complex modulus spectrum ( $M^{\prime \prime}$ vs $\left.M^{\prime}\right)$ of $\mathrm{Ba}_{3} \mathrm{Sr}_{2} \mathrm{DyTi}_{3} \mathrm{~V}_{7} \mathrm{O}_{30}$ at various temperatures. 
change in its shape with rise in temperature suggesting a change in the value of capacitance of the material with temperature.

Variation of real $\left(M^{\prime}\right)$ and imaginary $\left(M^{\prime \prime}\right)$ parts of the electric modulus as a function of frequency at various temperatures have been shown in figure 5. It is evident from figure 5(a) that for each temperature, $M^{\prime}$ reaches a constant value at higher frequencies. Also, at lower frequencies, $M^{\prime}$ approaches to zero, confirming the presence of an appreciable electrode and/or ionic polarization in temperature studied. The value of $M^{\prime}$ increases from the low frequency towards a high frequency limit and the dispersion shifts to high frequency as temperature increases. Variation of $M^{\prime \prime}$ with frequency (shown in figure 5(b)) at different temperatures reveals that as frequency increases $M^{\prime \prime}$ increases and reach to a maximum peak value at a particular frequency for all the temperatures. The trend in $M^{\prime \prime}$ continues with an exception that two peaks were observed below $T_{\mathrm{c}}$, whereas only one peak was observed above $T_{\mathrm{c}}$. The peak value of $M^{\prime \prime}$ for temperatures above $300^{\circ} \mathrm{C}$ is much larger compared to that of the same at transition temperature. The value of $M^{\prime \prime}$ increases with increase in frequency and takes a peak
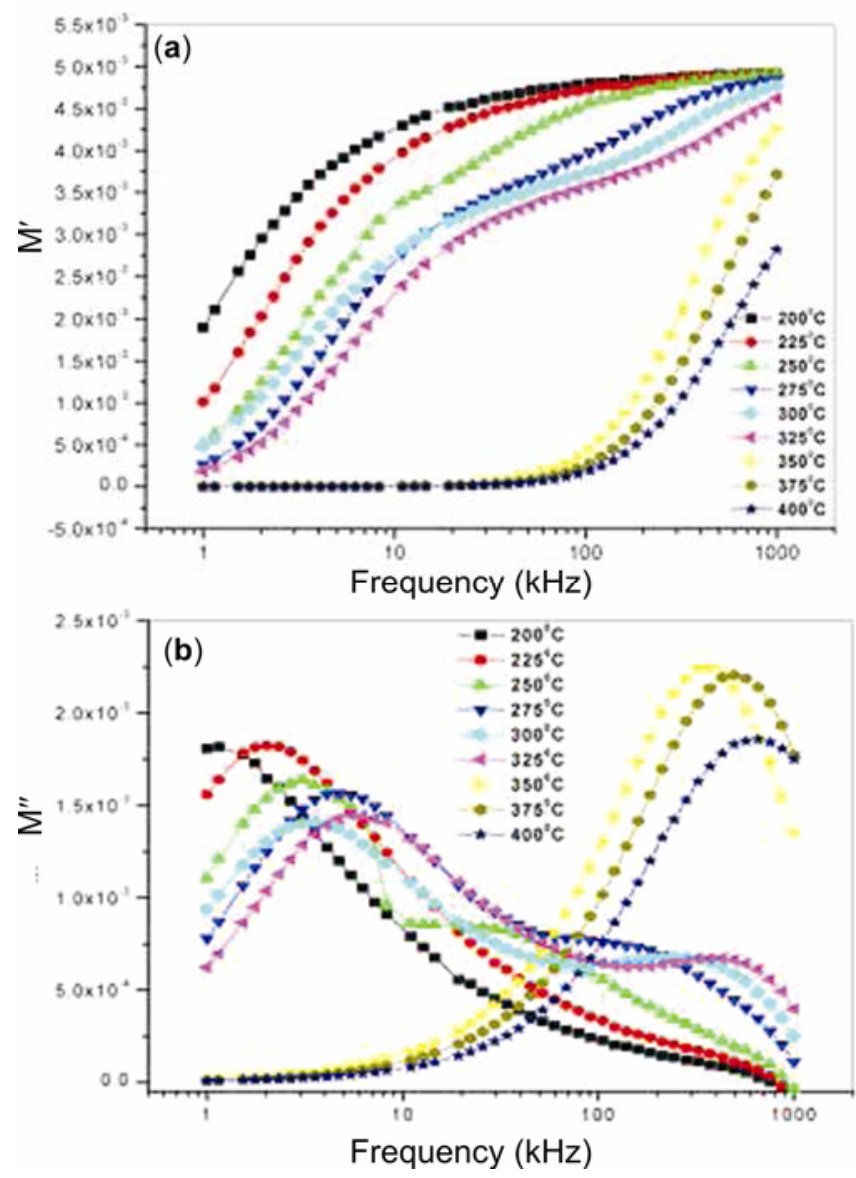

Figure 5. Variation of (a) real part of complex modulus $\left(M^{\prime}\right)$ and (b) imaginary part of complex modulus $\left(M^{\prime \prime}\right)$ of $\mathrm{Ba}_{3} \mathrm{Sr}_{2} \mathrm{DyTi}_{3} \mathrm{~V}_{7} \mathrm{O}_{30}$ with frequency. value at a particular frequency. With further increase in frequency, $M^{\prime \prime}$ decreases. Similar trend has been found at all its temperatures. However, $M^{\prime \prime}$ peak values shift towards higher frequency side. In a relaxation system, one can determine the most probable relaxation time $(\tau)$ from the position of the loss peak in $Z^{\prime \prime}$ or $M^{\prime \prime}$ with frequency plots according to the relation: $\tau=1 / \omega=1 / 2 \pi f_{\mathrm{r}}$. The variation of relaxation time $(\tau)$ with reciprocal of temperature $1 / T\left(\mathrm{~K}^{-1}\right)$ of BSDTV at high temperatures is shown in figure 6 and inset. This graph follows the Arrhenius relation, $\tau=\tau_{0} \exp \left(-E_{\mathrm{a}} / K_{\mathrm{B}} T\right)$, where the symbols have their usual meanings. The relaxation time is related to thermally activated process. The activation energy of the compound, calculated from above equation, is given in figure 6 . With the help of modulus plot, variation of $\tau$ with temperature is shown in figure 6 (inset). The value of the activation energy obtained from the slope of $\log (\tau)$ against $10^{3} T^{-1}$ curve is given in this figure. It is clear that the activation energy of the compound (calculated from loss and modulus spectrum) is nearly same. It suggests that the relaxation process may be attributed due to the same type of charge carriers. The scaling behaviour of the sample was studied by plotting normalized parameters (i.e. $M^{\prime \prime} / M_{\max }^{\prime \prime}$ versus $\log \left(f / f_{\max }\right)$, where $f_{\max }$ is frequency corresponding to $\left.M_{\max }^{\prime \prime}\right)$ at different temperatures (figure 7). The coincidence of all the curves of different temperatures into a single master curve indicates temperature independence of dynamic processes (Saha and Sinha 2002). These curves provide us with dielectric process occurring in the material and the magnitude of mismatch between the peaks.

\subsection{Conductivity analysis}

Electrical conductivity ( $\left.\sigma_{\text {d.c. }}\right)$ is a thermally activated process and follows the Arrhenius law. The activation

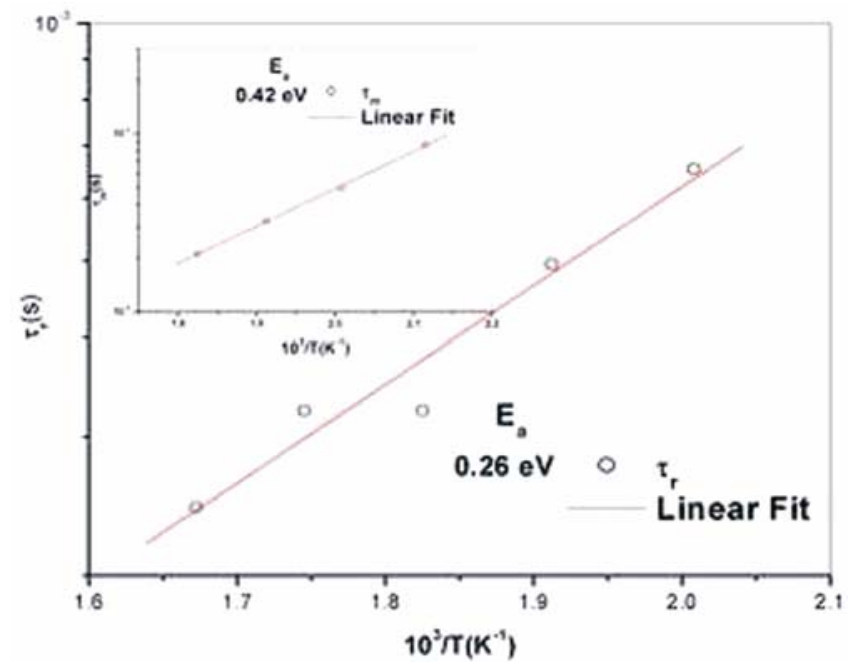

Figure 6. Variation of relaxation time with $10^{3} / T$ of $\mathrm{Ba}_{3} \mathrm{Sr}_{2} \mathrm{DyTi}_{3} \mathrm{~V}_{7} \mathrm{O}_{30}$ calculated from impedance spectrum ( $Z^{\prime \prime}$ vs frequency), and modulus spectrum ( $M^{\prime \prime}$ vs frequency) (inset). 
energy for conduction $\left(E_{\mathrm{a}}\right)$ of grains could be calculated from the slope of the straight line obtained from $\log \sigma_{\text {d.c. }}$ vs $1 / T$ plot. Figure 8 shows the Arrhenius plot of the d.c. conductivity evaluated from the impedance plots of BSDTV sample as a function of temperature, and the corresponding activation energy is found to be $0.41 \mathrm{eV}$. This value is same as calculated from modulus spectrum (figure 6 (inset)). It may be interpreted as the motion of oxygen vacancies, and are consistent with those obtained by the present impedance studies and also with earlier reports (Chen et al 1997).

Figure 9 shows frequency dependence of a.c. conductivity $\left(\sigma_{\text {a.c. }}\right)$ at various temperatures. It can be seen that at low frequencies and high temperatures we observe plateaus of $\sigma_{\text {a.c. }}$ (i.e. frequency independent values of conductivity) which corresponds to the d.c. conductivity. The

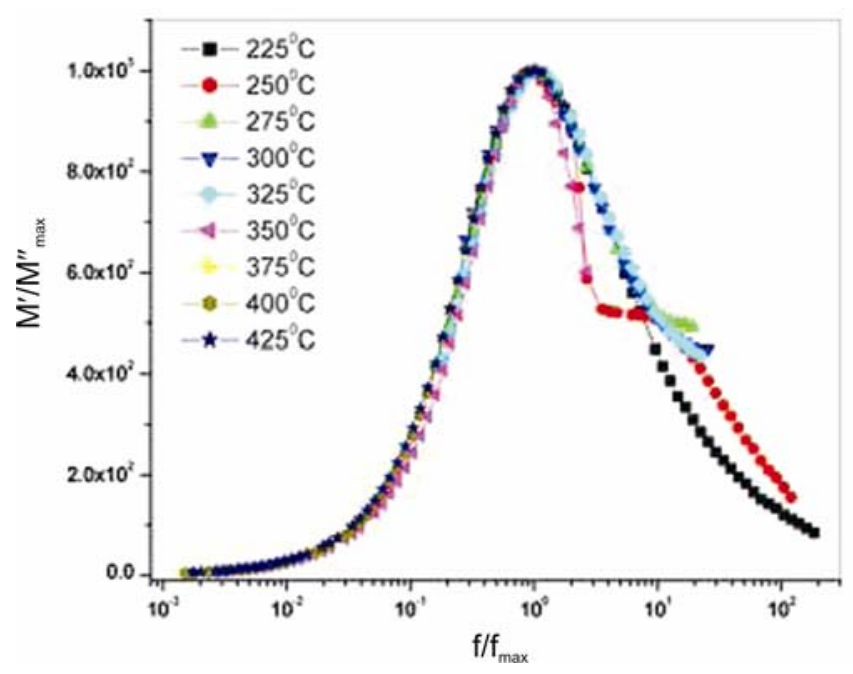

Figure 7. Variation of $M^{\prime \prime} / M_{\max }^{\prime \prime}$ with $f / f_{\max }$ of $\mathrm{Ba}_{3} \mathrm{Sr}_{2} \mathrm{Dy}$ $\mathrm{Ti}_{3} \mathrm{~V}_{7} \mathrm{O}_{30}$.

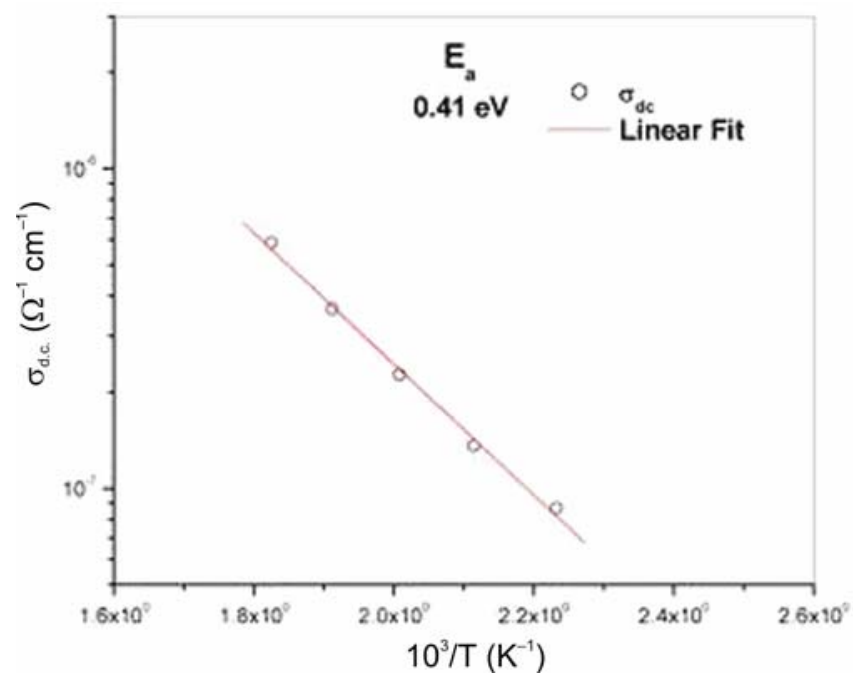

Figure 8. Variation of $\sigma_{\text {d.c. }}$ with $10^{3} / T$ of $\mathrm{Ba}_{3} \mathrm{Sr}_{2} \mathrm{DyTi}_{3} \mathrm{~V}_{7} \mathrm{O}_{30}$. observed frequency dependent conductivity can be described by the equation (Jonscher 1983)

$$
\sigma(\omega)=\sigma_{\text {d.c. }}+A \omega^{n},
$$

where $n$ is the frequency exponent in the range of $0<n<1, A$ and $n$ are thermally activated quantities, hence electrical conduction is a thermally activated process. According to Jonscher (1977), the origin of the frequency dependence of conductivity lies in the relaxation phenomena arising due to mobile charge carriers. When mobile charge carriers hop onto a new site from its original position, it remains in a state of displacement between two potential energy minima, which includes contributions from other mobile defects. After a sufficiently long time, the defect could relax until the two minima in lattice potential energy coincide with the lattice site.

\section{Conclusions}

The polycrystalline sample of BSDTV was prepared by a high-temperature solid-state-reaction route. Preliminary X-ray analysis confirms the orthorhombic crystal structure at room temperature. The surface morphology of the compound is studied through SEM, which gives the average grain size as the order of $1-2 \mu \mathrm{m}$. From the impedance and modulus spectroscopic studies the material showed relaxation effects which are non-Debye type. The relaxation frequencies shifted to higher frequency side with increase in temperature. The complex impedance plots reveal the main contribution of bulk in it. The variation of d.c. conductivity (bulk) as a function of temperature demonstrates that the compound exhibits Arrhenius type of electrical conductivity. Polarons at lower temperatures

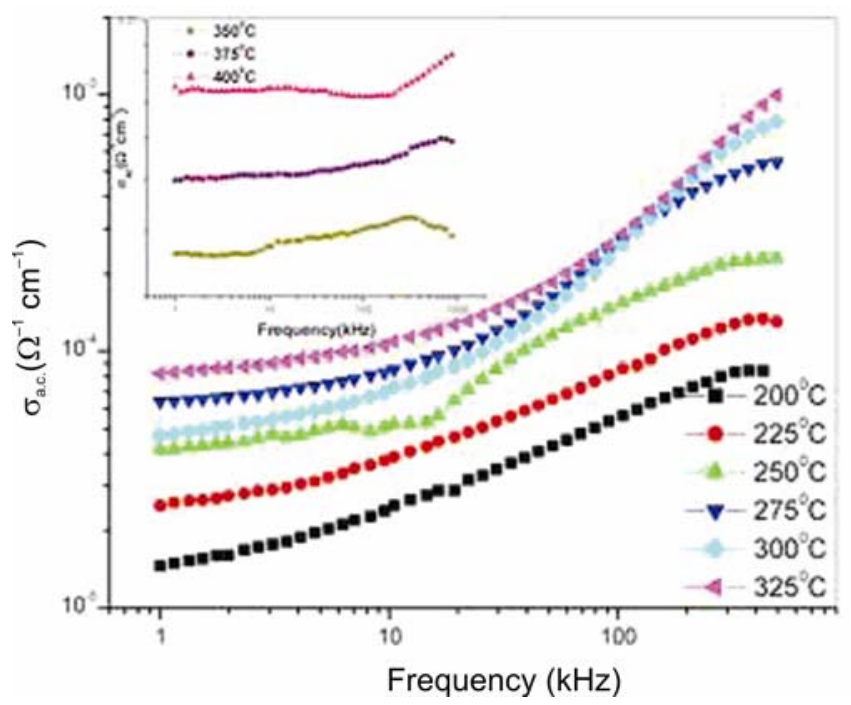

Figure 9. Variation of $\sigma_{\text {a.c. }}$ with frequency at different temperatures of $\mathrm{Ba}_{3} \mathrm{Sr}_{2} \mathrm{DyTi}_{3} \mathrm{~V}_{7} \mathrm{O}_{30}$ 
and singly ionized oxygen vacancies at higher temperatures also contribute to the conduction process in the material.

\section{References}

Chen T C, Thio C and Desu S B 1997 J. Mater. Res. 122628

Choudhary R N P, Pradhan D K, Tirado C M, Bonilla G E and Katiyar R S 2006 J. Appl. Phys. 1001

Choudhary R N P, Pradhan D K, Tirado C M, Bonilla G E and Katiyar R S 2007 Phys. Status Solidi (b) 2442254

Jiang W, Cao W, Yi X and Chen H 2005 J. Appl. Phys. 97 094106/1-4

Jonscher A K 1977 Nature 267673

Jonscher A K 1983 Dielectric relaxation in solids (London: Chelsea Dielectric Press)

Klug H P 1974 X-ray diffraction procedures for polycrystalline and amorphous materials (ed.) L E Alexander (New York: Wiley-Interscience)
MacDonald J R 1987 Impedance spectroscopy (New York: Wiley)

POWDMULT: An interactive powder diffraction data interpretations and indexing Program Version 2.1, E. WU School of Physical Sciences, Flinder University of South Australia Bradford Park, SA 5042, Australia

Ranga Raju M R and Choudhary R N P 2003 Mater. Lett. 57 2980

Saha S and Sinha T P 2002 Phys. Rev. B65 134103

Sahoo P S, Panigrahi A, Patri S K and Choudhary R N P 2008a Mod. Phys. Lett. B22 2999

Sahoo P S, Panigrahi A, Patri S K and Choudhary R N P 2008b CEJP (in press)

Singh K S, Sati R and Choudhary R N P 1992 J. Mater. Sci. Lett. 11788

Stenger C G F and Burggraaf A J 1980 J. Phys. Chem. Solids 4117

Suman C K, Prasad K and Choudhary R N P 2005 Adv. Appl. Ceram. 104294 